\title{
Ranking of fuzzy numbers based on alpha-distance
}

\author{
S. Khezerloo ${ }^{1}$ T. Allahviranloo ${ }^{2}$ M. Khezerloo ${ }^{1}$ \\ ${ }^{1}$ Young Researcher Club, Ardabil Branch, Islamic Azad University, Ardabil, Iran. \\ ${ }^{2}$ Department of Mathematics, Science and Research Branch, Islamic Azad University, Tehran, Iran
}

\begin{abstract}
This paper presents a new approach to compare fuzzy numbers using $\alpha$-distance. Initially, the metric distance on the interval numbers based on the convex hull of the endpoints is proposed and it is extended to fuzzy numbers. All the properties of the $\alpha$-distance are proved in details. Finally, the ranking of fuzzy numbers by the $\alpha$-distance is discussed. In addition, the proposed method is compared with some known ones, the validity of the new method is illustrated by applying its to several group of fuzzy numbers.
\end{abstract}

Keywords: Metric Distance; Fuzzy Numbers; Ranking.

\section{Introduction}

The concept of fuzzy numbers and arithmetic operations with these numbers were first introduced and investigated by Zadeh [3]. Additional related material can be found in $[1,10,11]$. Many researchers have developed methods to compare and to rank fuzzy numbers, too $[9,13,14,16,19]$. Cheng [4] has proposed some methods, and recently many ranking methods have been presented by Ma, Kandel and Friedman [12] and Chu and Tsao [15] . In [18] a fuzzy distance measure for Gaussian type fuzzy numbers is defined. In this study a new metric distance on fuzzy numbers is introduced and then it is used for ranking fuzzy numbers by comparison with two crisp numbers $\max (M)$ and $\min (m)$.

The rest of this paper is organized as follows: Section 2 contains preliminaries on fuzzy concepts. A metric distance measure for interval numbers with its properties is introduced in Section 3 . Then, in Section 4, $\alpha$-distance for fuzzy numbers is defined and its properties are discussed in detail. In the Section 5, we use a procedure for ranking fuzzy numbers based on the $\alpha$-distance. For comparing the proposed ranking method with some other approaches, some numerical examples are provided in Section 6. Finally, the paper ends with conclusions in Section 7.

\section{Preliminaries}

A fuzzy set $\tilde{A}=\left(a_{1}, a_{2}, a_{3}, a_{4}\right)$ is a generalized left right fuzzy numbers (GLRFN) of Dubois and Prade $[8,16]$, if its membership function satisfies the following:

$$
\mu_{\tilde{A}}(x)= \begin{cases}L\left(\frac{a_{2}-x}{a_{2}-a_{1}}\right), & a_{1} \leq x \leq a_{2}, \\ 1, & a_{2} \leq x \leq a_{3}, \\ R\left(\frac{x-a_{3}}{a_{4}-a_{3}}\right), & a_{3} \leq x \leq a_{4}, \\ 0, & \text { otherwise }\end{cases}
$$

where $\mathrm{L}$ and $\mathrm{R}$ are strictly decreasing functions defined on $[0,1]$ and satisfying the conditions:

$$
\begin{array}{lll}
L(t)=R(t)=1 & \text { if } \quad t \leq 0 \\
L(t)=R(t)=0 & \text { if } \quad t \geq 1
\end{array}
$$

For $a_{2}=a_{3}$, we have the classical definition of left right fuzzy numbers (LRFN) of Dubois and Prade $[8,16]$. Trapezoidal fuzzy numbers $(\operatorname{TrFN})$ are special cases of GLRFN with $L(t)=R(t)=1-t$. Triangular fuzzy numbers (TFN) are also special cases of GLRFN with $L(t)=R(t)=1-t$ and $a_{2}=a_{3}$. A GLRFN $\tilde{A}$ is denoted as $\tilde{A}=\left(a_{1}, a_{2}, a_{3}, a_{4}\right)_{L R}$ and an $\alpha$-level interval of fuzzy number $\tilde{A}$ as:

$$
\begin{aligned}
& {[\tilde{A}]^{\alpha}=\left[A_{l}(\alpha), A_{r}(\alpha)\right]} \\
& =\left[a_{2}-\left(a_{2}-a_{1}\right) L_{A}^{-1}(\alpha), a_{3}+\left(a_{4}-a_{3}\right) R_{A}^{-1}(\alpha)\right]
\end{aligned}
$$

If $\tilde{A}$ and $\tilde{B}$ are two GLRFN then:

$$
\tilde{A}+\tilde{B}=\tilde{C} \quad \text { if and only if }[\tilde{A}]^{\alpha}+[\tilde{B}]^{\alpha}=[\tilde{C}]^{\alpha}
$$

and

$$
\tilde{A} \cdot \tilde{B}=\tilde{C} \quad \text { if and only if }[\tilde{A}]^{\alpha} \cdot[\tilde{B}]^{\alpha}=[\tilde{C}]^{\alpha}
$$

where for interval numbers, we have

$$
[a, b]+[c, d]=[a+c, b+d]
$$

and

$[a, b] \cdot[c, d]=[\min \{a c, a d, b c, b d\}, \max \{a c, a d, b c, b d\}]$

\section{Distance for intervals}

Let $f(x)=(b-a) x+a$ and $g(x)=(d-c) x+c$ where $x \in[0,1]$. The distance between two intervals $[a, b]$ and $[c, d], a \leq b, c \leq d$, is denoted by $d_{I}^{(p)}([a, b],[c, d])$ such that:

$$
d_{I}^{(p)}([a, b],[c, d])=\left(D_{I}^{(p)}([a, b],[c, d])\right)^{\frac{1}{p}}
$$


and

$$
D_{I}^{(p)}([a, b],[c, d])=\|f(x)-g(x)\|_{L_{p}}^{p}
$$

where $\|$. $\|$ is the usual norm in the $L_{p}$ space on $[0,1](p>1)$.

This distance takes into account every point in both intervals with the convex combination of the lower and upper bound values of the both intervals. It is different from most of the existing distance measures for interval numbers (Bardossy et al.[1], Diamond [5], Diamond and Korner [6], and Diamond and Tanaka [7]). Tran and Duckstein [16] proposed a distance measure for intervals which also considers every point of the two intervals. But it was not a metric distance because $d([a, b],[a, b]) \neq 0$. Now we show that our proposed distance is a metric distance on interval numbers.

\subsection{Metric properties}

This proposed distance measure satisfies the following metric properties:

1. $d_{I}^{(p)}([a, b],[c, d]) \geq 0$

2. $[a, b]=[c, d]$ if and only if $d_{I}^{(p)}([a, b],[c, d])=0$

3. $d_{I}^{(p)}([a, b],[c, d])=d_{I}^{(p)}([c, d],[a, b])$

4. $d_{I}^{(p)}([a, b],[c, d])+d_{I}^{(p)}([c, d],[e, z]) \quad \geq$ $d_{I}^{(p)}([a, b],[e, z])$

The proofs follow immediately from the properties of the $L_{p}-$ norm.

\subsection{Other properties of $d_{I}^{(p)}$}

This distance satisfies the following properties if $a, b, c, d, e$ and $z$ are real numbers:

Proposition 1. If $\lambda \geq 0$, then

$$
d_{I}^{(p)}([\lambda a, \lambda b],[\lambda c, \lambda d])=|\lambda| d_{I}^{(p)}([a, b],[c, d])
$$

\section{Proposition 2.}

$d_{I}^{(p)}([a+\lambda, b+\lambda],[c+\lambda, d+\lambda])=d_{I}^{(p)}([a, b],[c, d])$

\section{Proposition 3.}

$$
d_{I}^{(p)}(a, b)=|a-b|
$$

(both intervals collapse to a single point).

Proposition 4. If $p=2$ then

$$
\sqrt{\frac{1}{3}\left((a-c)^{2}+(b-d)^{2}+(a-c)(b-d)\right)}
$$

Proposition 5. If $p=2$ then

$$
\begin{aligned}
& d_{I}^{(p)}([a, b], c)= \\
& \sqrt{\frac{1}{3}\left((a-c)^{2}+(b-c)^{2}+(a-c)(b-c)\right)}
\end{aligned}
$$

(one of the intervals collapses to a single point).

Proposition 6. $\frac{a+b}{2}$ is the nearest number to $[a, b]$ by using this metric.

Proposition 7. If $p=2$ then

$$
d_{I}^{(p)}([a, b], 0)=\sqrt{\frac{1}{3}\left(a^{2}+b^{2}+a b\right)}
$$

is equal to the distance measure of Tran and Duckstein [16].

The proofs of Propositions 1, 2, 3, 4, 5, 6 and 7 are clear.

\section{Proposition 8.}

$$
d_{I}^{(p)}([a, b]+[c, d],[a, b]+[e, z])=d_{I}^{(p)}([c, d],[e, z])
$$

\section{Proof:}

$$
\begin{aligned}
& d_{I}^{(p)}([a, b]+[c, d],[a, b]+[e, z]) \\
& =d_{I}^{(p)}([a+c, b+d],[a+e, b+z]) \\
& =\left(\int_{0}^{1}((b+d-a-c) x+a+c)\right. \\
& \left.\quad-((b+z-a-e) x+a+e)^{p} d x\right)^{\frac{1}{p}} \\
& =\left(\int_{0}^{1}((d-c) x+c)-((z-e) x+e)^{p} d x\right)^{\frac{1}{p}} \\
& =d_{I}^{(p)}([c, d],[e, z])
\end{aligned}
$$

Definition 1. If $[a, b]$ and $[c, d]$ are two intervals and $M$ is a crisp number, then by (8) and (9), we have

$$
d_{I}^{(p)}([a, b] \cdot[c, d], M)=\left(D_{I}^{(p)}([a, b] \cdot[c, d], M)\right)^{\frac{1}{p}}
$$

where

$D_{I}^{(p)}([a, b] .[c, d], M)=\int_{0}^{1} D_{I}^{(p)}([a, b] .((d-c) x+c), M) d x$

If $c=d=1$ then

$$
d_{I}^{(p)}([a, b] \cdot[c, d], M)=d_{I}^{(p)}([a, b], M)
$$

\section{Metric for fuzzy numbers}

$\alpha$-distance between two GLRFNs $\tilde{A}$ and $\tilde{B}$ by using the metric defined in Section 3, can be defined as:

$$
d_{F}^{(p)}(\tilde{A}, \tilde{B})=\left(D_{F}^{(p)}(\tilde{A}, \tilde{B})\right)^{\frac{1}{p}}
$$

such that

$$
D_{F}^{(p)}(\tilde{A}, \tilde{B})=\sup _{\alpha \in[0,1]} D_{I}^{(p)}\left([\tilde{A}]^{\alpha},[\tilde{B}]^{\alpha}\right)
$$

It can be proved that $d_{F}^{(p)}(\tilde{A}, \tilde{B})$ is a metric on GLRFNs.

\subsection{The properties of $\alpha$-distance}

The proposed distance ' $\alpha$-distance' has the following metric properties:

1. $d_{F}^{(p)}(\tilde{A}, \tilde{B}) \geq 0$ 
2. $\tilde{A}=\tilde{B}$ if and only if $d_{F}^{(p)}(\tilde{A}, \tilde{B})=0$

3. $d_{F}^{(p)}(\tilde{A}, \tilde{B})=d_{F}^{(p)}(\tilde{B}, \tilde{A})$

4. $d_{F}^{(p)}(\tilde{A}, \tilde{B})+d_{F}^{(p)}(\tilde{B}, \tilde{C}) \geq d_{F}^{(p)}(\tilde{A}, \tilde{C})$

\section{Proofs:}

1. Since $D_{I}^{(p)}\left([\tilde{A}]^{\alpha},[\tilde{B}]^{\alpha}\right) \geq 0$ then $D_{F}^{(p)}(\tilde{A}, \tilde{B}) \geq$ 0 hence $d_{F}^{(p)}(\tilde{A}, \tilde{B}) \geq 0$.

2. We know that, $\tilde{A}=\tilde{B}$ if and only if $[\tilde{A}]^{\alpha}=[\tilde{B}]^{\alpha}$ for all $\alpha \in[0,1]$. But, since $d_{I}^{(p)}([.,],.[.,]$.$) is a$ metric distance, then $[\tilde{A}]^{\alpha}=[\tilde{B}]^{\alpha}$ if and only if $D_{I}^{(p)}\left([\tilde{A}]^{\alpha},[\tilde{B}]^{\alpha}\right)=0$ for all $\alpha \in[0,1]$. Hence, $\tilde{A}=\tilde{B}$ if and only if $D_{F}^{(p)}(\tilde{A}, \tilde{B})=0$ and then $d_{F}^{(p)}(\tilde{A}, \tilde{B})=0$.

3. From equations (13) and (14),

$$
\begin{aligned}
d_{F}^{(p)}(\tilde{A}, \tilde{B}) & =\left(\sup _{\alpha \in[0,1]} D_{I}^{(p)}\left([\tilde{A}]^{\alpha},[\tilde{B}]^{\alpha}\right)^{\frac{1}{p}}\right. \\
& =\left(\sup _{\alpha \in[0,1]} D_{I}^{(p)}\left([\tilde{B}]^{\alpha},[\tilde{A}]^{\alpha}\right)\right)^{\frac{1}{p}} \\
& =\left(D_{F}^{(p)}(\tilde{B}, \tilde{A})\right)^{\frac{1}{p}} \\
& =d_{F}^{(p)}(\tilde{B}, \tilde{A}) .
\end{aligned}
$$

4. From equations (13) and (14),

$$
\begin{aligned}
d_{F}^{(p)}(\tilde{A}, \tilde{C}) & =\left(D_{F}^{(p)}(\tilde{A}, \tilde{C})\right)^{\frac{1}{p}} \\
& =\left(\sup _{\alpha \in[0,1]}\left(D_{I}^{(p)}\left([A]^{\alpha},[C]^{\alpha}\right)\right)\right)^{\frac{1}{p}} \\
& =\sup _{\alpha \in[0,1]}\left(D_{I}^{(p)}\left([A]^{\alpha},[C]^{\alpha}\right)\right)^{\frac{1}{p}} \\
& =\sup _{\alpha \in[0,1]}\left(d_{I}^{(p)}\left([A]^{\alpha},[C]^{\alpha}\right)\right) \\
& \leq \sup _{\alpha \in[0,1]}\left(d_{I}^{(p)}\left([A]^{\alpha},[B]^{\alpha}\right)\right. \\
& \left.\quad+d_{I}^{(p)}\left([B]^{\alpha},[C]^{\alpha}\right)\right) \\
& \leq \sup _{\alpha \in[0,1]}\left(d_{I}^{(p)}\left([A]^{\alpha},[B]^{\alpha}\right)\right) \\
& +\sup _{\alpha \in[0,1]}\left(d_{I}^{(p)}\left([B]^{\alpha},[C]^{\alpha}\right)\right) \\
& =d_{F}^{(p)}(\tilde{A}, \tilde{B})+d_{F}^{(p)}(\tilde{B}, \tilde{C})
\end{aligned}
$$

Therefore, the proofs are completed.

\subsection{Other properties of $\alpha$-distance}

This distance satisfies the following properties:

Proposition 9. If $\lambda \geq 0$, then

$$
d_{F}^{(p)}(\lambda \tilde{E}, \lambda \tilde{F})=|\lambda| d_{F}^{(p)}(\tilde{E}, \tilde{F})
$$

\section{Proposition 10.}

$$
d_{F}^{(p)}(\tilde{E}+\lambda, \tilde{F}+\lambda)=d_{F}^{(p)}(\tilde{E}, \tilde{F})
$$

\section{Proposition 11.}

$$
d_{F}^{(p)}(\tilde{E}+\tilde{G}, \tilde{E}+\tilde{F})=d_{F}^{(p)}(\tilde{G}, \tilde{F})
$$

Proof: By considering Proposition (8)

$$
\begin{aligned}
& d_{F}^{(p)}(\tilde{E}+\tilde{G}, \tilde{E}+\tilde{F})=\left(D_{F}^{(p)}(\tilde{E}+\tilde{G}, \tilde{E}+\tilde{F})\right)^{\frac{1}{p}} \\
& =\left(\sup _{\alpha \in[0,1]} D_{I}^{(p)}\left([\tilde{E}]^{\alpha}+[\tilde{G}]^{\alpha},[\tilde{E}]^{\alpha}+[\tilde{F}]^{\alpha}\right)\right)^{\frac{1}{p}} \\
& =\left(\sup _{\alpha \in[0,1]} D_{I}^{(p)}\left([\tilde{G}]^{\alpha},[\tilde{F}]^{\alpha}\right)\right)^{\frac{1}{p}} \\
& =\left(D_{F}^{(p)}(\tilde{G}, \tilde{F})\right)^{\frac{1}{p}} \\
& =d_{F}^{(p)}(\tilde{G}, \tilde{F})
\end{aligned}
$$

Then the proof is completed.

Proposition 12.

$$
d_{F}^{(p)}(a, b)=|a-b|
$$

(both fuzzy numbers collapse to a single point).

\section{Proposition 13.}

$$
d_{F}^{(p)}([a, b],[c, d])=d_{I}^{(p)}([a, b],[c, d])
$$

(both fuzzy numbers collapse to an interval).

Definition 2. If $\tilde{A}$ and $\tilde{B}$ are two fuzzy numbers and $M$ is a crisp number then by (13), (14)

$$
d_{F}^{(p)}(\tilde{A} . \tilde{B}, M)=\left(D_{F}^{(p)}(\tilde{A} . \tilde{B}, M)\right)^{\frac{1}{p}}
$$

where

$$
D_{F}^{(p)}(\tilde{A} \cdot \tilde{B}, M)=\sup _{\alpha \in[0,1]} D_{I}^{(p)}\left([A]^{\alpha} \cdot[B]^{\alpha}, M\right) d \alpha
$$

Then regarding (12)

$$
\begin{aligned}
& D_{F}^{(p)}(\tilde{A} . \tilde{B}, M)=\sup _{\alpha \in[0,1]} \\
& \int_{0}^{1} D_{I}^{(p)}\left([A]^{\alpha} \cdot\left(\left(B_{l}(\alpha)-B_{r}(\alpha)\right) x+B_{r}(\alpha)\right), M\right) d x
\end{aligned}
$$

If $\tilde{B}=1$ then $d_{F}^{(p)}(\tilde{A} . \tilde{B}, M)=d_{F}^{(p)}(\tilde{A}, M)$.

\section{Ranking fuzzy numbers by $\alpha$-distance}

Ranking of fuzzy numbers has been studied by many researchers. Some researchers introduced distances and then compared fuzzy numbers with them $([2,4,16,19])$. Other researchers used the nearest symmetric (nonsymmetric) triangular fuzzy number or symmetric (nonsymmetric) trapezoidal fuzzy number for comparing fuzzy numbers, $([9,13])$. Each method has its own shortcoming. In this section, we propose an approach for ranking two fuzzy numbers $\tilde{A}$ and $\tilde{B}$ based on the $\alpha$ distance. For ranking two fuzzy numbers $\tilde{A}$ and $\tilde{B}$, if $\sup (\operatorname{supp}(\tilde{A}))<\inf (\operatorname{supp}(\tilde{B}))$, it is evident that 
$\tilde{A}<\tilde{B}$ and that the degree of $\tilde{A}<\tilde{B}$ is 1 and the degree of $\tilde{A}>\tilde{B}$ is 0 ; therefore, we consider two fuzzy numbers $\tilde{A}$ and $\tilde{B}$ such that $\operatorname{supp}(\tilde{A}) \cap \operatorname{supp}(\tilde{B}) \neq \phi$. Tran and Duckstein [16] proposed a similar distance for ranking but it was not a metric distance because $d([a, b],[a, b]) \neq 0$. The proposed method in this paper for ranking two fuzzy numbers $\tilde{A}$ and $\tilde{B}$ is based on the comparison of the degree of distance between the fuzzy numbers $\tilde{A}$ and $\tilde{B}$ and $\operatorname{crisp} \max (M)$ and $\operatorname{crisp} \min (m)$ where:

$$
\left\{\begin{array}{c}
M \geq \max (\operatorname{supp} \tilde{A} \cup \operatorname{supp} \tilde{B}) \\
\& \\
m \leq \min (\operatorname{supp} \tilde{A} \cup \operatorname{supp} \tilde{B})
\end{array}\right.
$$

We denote the degree of distance between $\tilde{A}$ and crisp numbers $\max (M)$ and $\min (m)$ by $\gamma_{d}^{(p)}(\tilde{A}, M)$ and $\gamma_{d}^{(p)}(\tilde{A}, m)$, respectively, which are defined as follows:

$$
\begin{aligned}
\gamma_{d}^{(p)}(\tilde{A}, M) & =\frac{d_{F}^{(p)}(\tilde{A}, M)}{d_{F}^{(p)}(\tilde{A}, M)+d_{F}^{(p)}(\tilde{A}, m)} \\
\gamma_{d}^{(p)}(\tilde{A}, m) & =\frac{d_{F}^{(p)}(\tilde{A}, m)}{d_{F}^{(p)}(\tilde{A}, M)+d_{F}^{(p)}(\tilde{A}, m)}
\end{aligned}
$$

such that $d_{F}^{(p)}(\tilde{A}, M)$ and $d_{F}^{(p)}(\tilde{A}, m)$ in (13) and (14) are the distances between the fuzzy number $\tilde{A}$ and crisp numbers $\max (M)$ and $\min (m)$, respectively.

\section{Proposition 14.}

$$
\gamma_{d}^{(p)}(\tilde{A}, M)+\gamma_{d}^{(p)}(\tilde{A}, m)=1
$$

\section{Proposition 15.}

$$
\gamma_{d}^{(p)}(\tilde{A}, M) \geq \gamma_{d}^{(p)}(\tilde{B}, M)
$$

if only if

$$
\gamma_{d}^{(p)}(\tilde{A}, m) \leq \gamma_{d}^{(p)}(\tilde{B}, m)
$$

Proof: By Proposition 14, the proof is clear.

Proposition 16. If $M^{\prime}>M$ and

$$
\gamma_{d}^{(p)}(\tilde{A}, M) \geq \gamma_{d}^{(p)}(\tilde{B}, M)
$$

then

$$
\gamma_{d}^{(p)}\left(\tilde{A}, M^{\prime}\right) \geq \gamma_{d}^{(p)}(\tilde{B}, M)
$$

Proof: The proof is clear.

Proposition 17. If $m^{\prime}<m$ and

$$
\gamma_{d}^{(p)}(\tilde{A}, m) \leq \gamma_{d}^{(p)}(\tilde{B}, m)
$$

then

$$
\gamma_{d}^{(p)}\left(\tilde{A}, m^{\prime}\right) \leq \gamma_{d}^{(p)}(\tilde{B}, m)
$$

Proof: The proof is clear.

Definition 3. The ranking method is as follows: (a):

$$
\tilde{A} \precsim \tilde{B} \Leftrightarrow\left\{\begin{array}{c}
\gamma_{d}^{(p)}(\tilde{A}, M) \geq \gamma_{d}^{(p)}(\tilde{B}, M) \\
\text { or } \\
\gamma_{d}^{(p)}(\tilde{A}, m) \leq \gamma_{d}^{(p)}(\tilde{B}, m)
\end{array}\right.
$$

such that the degree of ranking is defined as:

$$
\begin{aligned}
\gamma_{(\lambda M+(1-\lambda) m)}^{(\tilde{A}<\tilde{B})} & =\frac{\lambda \cdot \gamma_{d}^{(p)}(\tilde{A}, M)}{\gamma_{d}^{(p)}(\tilde{A}, M)+\gamma_{d}^{(p)}(\tilde{B}, M)} \\
& +\frac{(1-\lambda) \cdot \gamma_{d}^{(p)}(\tilde{B}, m)}{\gamma_{d}^{(p)}(\tilde{A}, m)+\gamma_{d}^{(p)}(\tilde{B}, m)}, \\
& \lambda \in[0,1]
\end{aligned}
$$

$\lambda$ is chosen according to the decision-maker's idea. If $\lambda=0$, the ranking method is as presented in (22); if $\lambda=1$, the ranking method is as provided in (21) and if $0<\lambda<1$, the ranking method works with crisp $\min (m)$ and crisp $\max (M)$.

(b):

$$
\tilde{A}=\tilde{B} \quad \Leftrightarrow \quad d_{F}^{(p)}(\tilde{A}, \tilde{B})=0
$$

then the degree of ranking is defined as follows:

$$
\gamma_{(\lambda . M+(1-\lambda) m)}^{(\tilde{A}=\tilde{B})}=1
$$

and

$$
\gamma_{(\lambda . M+(1-\lambda) m)}^{(\tilde{A}>\tilde{B})}=\gamma_{(\lambda . M+(1-\lambda) m)}^{(\tilde{A}<\tilde{B})}=0
$$

(c):

$$
\tilde{A} \approx \tilde{B} \Leftrightarrow\left\{\begin{array}{c}
\gamma_{d}^{(p)}(\tilde{A}, M)=\gamma_{d}^{(p)}(\tilde{B}, M)=\frac{1}{2} \\
\& \\
\gamma_{d}^{(p)}(\tilde{A}, m)=\gamma_{d}^{(p)}(\tilde{B}, m)=\frac{1}{2}
\end{array}\right.
$$

In this case, we have the maximum ambiguity for ranking, therefore the degree of ranking is defined as follows:

$$
\begin{aligned}
\gamma_{(\lambda . M+(1-\lambda) m)}^{(\tilde{A}=\tilde{B})} & =\gamma_{(\lambda . M+(1-\lambda) m)}^{(\tilde{A}>\tilde{B})} \\
& =\gamma_{(\lambda . M+(1-\lambda) m)}^{(\tilde{A}<\tilde{B})} \\
& =\frac{1}{2}
\end{aligned}
$$

In this method, for ranking $n$ fuzzy numbers $\tilde{A}_{1}, \ldots, \tilde{A}_{n}$ we compare the degree of distance between the fuzzy numbers and any crisp $\max (M)$ and any $\operatorname{crisp} \min (m)$ where:

$$
\left\{\begin{array}{c}
M \geq \max \left(\cup_{i=1}^{n} \operatorname{supp} \tilde{A}_{i}\right) \\
\& \\
m \leq \min \left(\cup_{i=1}^{n} \operatorname{supp} \tilde{A}_{i}\right)
\end{array}\right.
$$

If we add $\tilde{C}$ to the set, then $M$ and $m$, as well as, may change, but regarding Proposition, (16) 
and (17), there will be no change in the ranking of $\tilde{A}_{1}, \ldots, \tilde{A}_{n}$.

\section{Proposition 18.}

$\gamma_{(\lambda M+(1-\lambda) m)}^{(\tilde{A}<\tilde{B})}+\gamma_{(\lambda M+(1-\lambda) m)}^{(\tilde{A}>\tilde{B})}=1, \quad \lambda \in[0,1]$.

Proposition 19. If $\tilde{A} \precsim \tilde{B}$ and $\tilde{B} \precsim \tilde{C}$ then for each $\lambda \in[0,1]$,

$$
\begin{aligned}
& \gamma_{\left(\lambda . M^{\prime \prime}+(1-\lambda) m^{\prime \prime}\right)}^{(\tilde{A}<\tilde{\tilde{C}})} \geq \\
& \max \left\{\gamma_{(\lambda . M+(1-\lambda) m)}^{(\tilde{A}<\tilde{B})}, \quad \gamma_{\left(\lambda . M^{\prime}+(1-\lambda) m^{\prime}\right)}^{(\tilde{B}<\tilde{C})}\right\}
\end{aligned}
$$

where

$$
\begin{aligned}
& M \geq \max (\operatorname{supp} \tilde{A} \cup \operatorname{supp} \tilde{B}), \\
& m \leq \min (\operatorname{supp} \tilde{A} \cup \operatorname{supp} \tilde{B}), \\
& M^{\prime} \geq \max (\operatorname{supp} \tilde{B} \cup \operatorname{supp} \tilde{C}), \\
& m^{\prime} \leq \min (\operatorname{supp} \tilde{B} \cup \operatorname{supp} \tilde{C}), \\
& M^{\prime \prime} \geq \max \left(M, M^{\prime}\right), \quad m^{\prime \prime} \leq \min \left(m, m^{\prime}\right) .
\end{aligned}
$$

Now we consider the following reasonable properties for the ordering approaches on E (the set of fuzzy numbers)(see [17]).

A: For an arbitrary finite subset $\Lambda$ of $E$ and $\tilde{u} \in \Lambda$, $\tilde{u} \precsim \tilde{u}$.

Proof: The proof is evident by the definition of ranking.

B: For an arbitrary finite subset $\Lambda$ of $E$ and $(\tilde{u}, \tilde{v}) \in \Lambda^{2}, \tilde{u} \succsim \tilde{v}$ and $\tilde{u} \precsim \tilde{v}$, we should have $\tilde{u} \approx \tilde{v}$.

Proof: The proof is clear by the definition of ranking.

C: For an arbitrary finite subset $\Lambda$ of $E$ and $(\tilde{u}, \tilde{v}, \tilde{z}) \in \Lambda^{3}, \tilde{u} \precsim \tilde{v}$ and $\tilde{v} \precsim \tilde{z}$, we should have $\tilde{u} \precsim \tilde{z}$.

Proof: From (21), (22) and (23)

$$
\begin{gathered}
d_{F}^{(p)}(\tilde{u}, M) d_{F}^{(p)}(\tilde{v}, m) \geq d_{F}^{(p)}(\tilde{v}, M) d_{F}^{(p)}(\tilde{u}, m) \\
d_{F}^{(p)}\left(\tilde{v}, M^{\prime}\right) d_{F}^{(p)}\left(\tilde{z}, m^{\prime}\right) \geq d_{F}^{(p)}\left(\tilde{z}, M^{\prime}\right) d_{F}^{(p)}\left(\tilde{v}, m^{\prime}\right)
\end{gathered}
$$

where

$$
\begin{aligned}
& M^{\prime} \geq \max (\operatorname{supp} \tilde{v} \cup \operatorname{supp} \tilde{z}) \\
& m^{\prime} \leq \min (\operatorname{supp} \tilde{v} \cup \operatorname{supp} \tilde{z}) \\
& M \geq \max (\operatorname{supp} \tilde{u} \cup \operatorname{supp} \tilde{v}) \\
& m \leq \min (\operatorname{supp} \tilde{u} \cup \operatorname{supp} \tilde{v})
\end{aligned}
$$

It is sufficient to show that

$$
d_{F}^{(p)}\left(\tilde{u}, M^{\prime \prime}\right) d_{F}^{(p)}\left(\tilde{z}, m^{\prime \prime}\right) \geq d_{F}^{(p)}\left(\tilde{z}, M^{\prime \prime}\right) d_{F}^{(p)}\left(\tilde{u}, m^{\prime \prime}\right)
$$

where

$$
M^{\prime \prime} \geq \max \left\{M, M^{\prime}\right\}
$$

and

$$
m^{\prime \prime} \leq \min \left\{m, m^{\prime}\right\}
$$

Therefore

$$
\begin{aligned}
& d_{F}^{(p)}\left(\tilde{u}, M^{\prime \prime}\right) d_{F}^{(p)}\left(\tilde{z}, m^{\prime \prime}\right) \\
& \geq d_{F}^{(p)}\left(\tilde{v}, M^{\prime \prime}\right) d_{F}^{(p)}\left(\tilde{z}, m^{\prime \prime}\right) \\
& \geq d_{F}^{(p)}\left(\tilde{v}, m^{\prime \prime}\right) d_{F}^{(p)}\left(\tilde{z}, M^{\prime \prime}\right) \\
& \geq d_{F}^{(p)}\left(\tilde{u}, m^{\prime \prime}\right) d_{F}^{(p)}\left(\tilde{z}, M^{\prime \prime}\right)
\end{aligned}
$$

D: For an arbitrary finite subset $\Lambda$ of $E$ and $(\tilde{u}, \tilde{v}) \in \Lambda^{2}, \inf \operatorname{supp}(\tilde{u})>\operatorname{supsupp}(\tilde{v})$, we should have $\tilde{u} \succsim \tilde{v}$.

Proof: The proof is obvious by the definition of ranking.

$D^{\prime}:$ For an arbitrary finite subset $\Lambda$ of $E$ and $(\tilde{u}, \tilde{v}) \in \Lambda^{2}, \inf \operatorname{supp}(\tilde{u})>\operatorname{supsupp}(\tilde{v})$, we should have $\tilde{u} \succ \tilde{v}$.

Proof: The proof is clear by the definition of ranking.

E: Let $\Lambda$ and $\Lambda^{\prime}$ be two arbitrary finite subsets of $E$ in which $\tilde{u}$ and $\tilde{v}$ are in $\Lambda \cap \Lambda^{\prime}$. We obtain the ranking order $\tilde{u} \succeq \tilde{v}$ by $d_{F}$ on $\Lambda^{\prime}$ if and only if $\tilde{u} \succeq \tilde{v}$ by $d_{F}$ on $\Lambda$.

Proof: The proof is evident by the definition of ranking.

F: Let $\tilde{u}, \tilde{v}, \tilde{u}+\tilde{z}$ and $\tilde{v}+\tilde{z}$ be elements of $E$. If $\tilde{u} \precsim \tilde{v}$, then $\tilde{u}+\tilde{z} \precsim \tilde{v}+\tilde{z}$.

Proof: From (21), (22) and (23)

$$
d_{F}^{(p)}(\tilde{u}, M) d_{F}^{(p)}(\tilde{v}, m) \geq d_{F}^{(p)}(\tilde{v}, M) d_{F}^{(p)}(\tilde{u}, m)
$$

where

$$
\begin{aligned}
& M \geq \max (\text { supp } \tilde{u} \cup \operatorname{supp} \tilde{v}), \\
& m \leq \min (\operatorname{supp} \tilde{u} \cup \operatorname{supp} \tilde{v})
\end{aligned}
$$

It is sufficient to show that

$$
\begin{aligned}
& d_{F}^{(p)}\left(\tilde{u}+\tilde{z}, M+M^{\prime}\right) d_{F}^{(p)}\left(\tilde{v}+\tilde{z}, m+m^{\prime}\right) \geq \\
& d_{F}^{(p)}\left(\tilde{v}+\tilde{z}, M+M^{\prime}\right) d_{F}^{(p)}\left(\tilde{u}+\tilde{z}, m+m^{\prime}\right)
\end{aligned}
$$

where

$$
\begin{aligned}
& M^{\prime} \geq \max (\operatorname{supp} \tilde{z}), \\
& m^{\prime} \leq \min (\operatorname{supp} \tilde{z})
\end{aligned}
$$

It is obvious that

$$
d_{F}^{(p)}\left(\tilde{u}+\tilde{z}, M+M^{\prime}\right) \geq d_{F}^{(p)}\left(\tilde{v}+\tilde{z}, M+M^{\prime}\right)
$$


and

$$
d_{F}^{(p)}\left(\tilde{v}+\tilde{z}, m+m^{\prime}\right) \geq d_{F}^{(p)}\left(\tilde{u}+\tilde{z}, m+m^{\prime}\right)
$$

$F^{\prime}:$ Let $\tilde{u}, \tilde{v}, \tilde{u}+\tilde{z}$ and $\tilde{v}+\tilde{z}$ be elements of E. If $\tilde{u}>\tilde{v}$, then $\tilde{u}+\tilde{z}>\tilde{v}+\tilde{z}$ when $\tilde{z} \neq 0$.

Proof: If $\tilde{u}>\tilde{v}$ then

$$
\max (\operatorname{supp} \tilde{v})<\min (\operatorname{supp} \tilde{u})
$$

It is clear that if $\tilde{z} \neq 0$ then

$$
\max (\operatorname{supp}(\tilde{v}+\tilde{z}))<\min (\operatorname{supp}(\tilde{u}+\tilde{z}))
$$

hence,

$$
\tilde{u}+\tilde{z}>\tilde{v}+\tilde{z}
$$

G: Let $\tilde{u}, \tilde{v}, \tilde{u} \tilde{z}$ and $\tilde{v} \tilde{z}$ be elements of $E$ and

$$
\tilde{z} \geq 0(\min (\operatorname{supp} \tilde{z}) \geq 0)
$$

$\tilde{u} \precsim \tilde{v}$, implies $\tilde{u} \tilde{z} \precsim \tilde{v} \tilde{z}$.

Proof: It is sufficient to show that

$$
\begin{aligned}
& d_{F}^{(p)}\left(\tilde{u} \tilde{z}, M^{\prime} M\right) d_{F}^{(p)}\left(\tilde{v} \tilde{z}, m^{\prime} m\right) \geq \\
& d_{F}^{(p)}\left(\tilde{v} \tilde{z}, M^{\prime} M\right) d_{F}^{(p)}\left(\tilde{u} \tilde{z}, m^{\prime} m\right)
\end{aligned}
$$

where

$$
\begin{aligned}
& M^{\prime} \geq \max (\operatorname{supp} \tilde{z}) \\
& m^{\prime} \leq \min (\operatorname{supp} \tilde{z}) \\
& M \geq \max (\operatorname{supp} \tilde{u} \cup \operatorname{supp} \tilde{v}) \\
& m \leq \min (\operatorname{supp} \tilde{u} \cup \operatorname{supp} \tilde{v})
\end{aligned}
$$

It is sufficient to show that

$$
\left\{\begin{array}{l}
d_{F}^{(p)}\left(\tilde{u} \tilde{z}, M^{\prime} M, s\right) \geq d_{F}^{(p)}\left(\tilde{v} \tilde{z}, M^{\prime} M\right) \\
d_{F}^{(p)}\left(\tilde{v} \tilde{z}, m^{\prime} m\right) \geq d_{F}^{(p)}\left(\tilde{u} \tilde{z}, m^{\prime} m\right)
\end{array}\right.
$$

We demonstrate that the proofs of $d_{F}^{(p)}\left(\tilde{u} \tilde{z}, M^{\prime} M\right) \geq d_{F}^{(p)}\left(\tilde{v} \tilde{z}, M^{\prime} M\right)$ and that of $d_{F}^{(p)}\left(\tilde{v} \tilde{z}, m^{\prime} m\right) \geq d_{F}^{(p)}\left(\tilde{u} \tilde{z}, m^{\prime} m\right)$ are similar. By (19)

$$
\begin{aligned}
& D_{F}^{(p)}\left(\tilde{u} \tilde{z}, M^{\prime} M\right)=\sup _{\alpha \in[0,1]} \\
& \int_{0}^{1} D_{I}^{(p)}\left([u]^{\alpha} \cdot\left(\left(z_{l}(\alpha)-z_{r}(\alpha)\right) x+z_{r}(\alpha)\right), M^{\prime} M\right) d x
\end{aligned}
$$

Considering (13), it is sufficient to show that

$$
D_{F}^{(p)}\left(\tilde{u} \tilde{z}, M^{\prime} M\right) \geq D_{F}^{(p)}\left(\tilde{v} \tilde{z}, M^{\prime} M\right)
$$

It is clear that

$$
\frac{D_{F}^{(p)}\left(\tilde{u} \tilde{z}, M^{\prime} M\right)}{D_{F}^{(p)}\left(\tilde{v} \tilde{z}, M^{\prime} M\right)} \geq 1
$$

if

$\frac{\sup _{\alpha \in[0,1]} D_{I}^{(p)}\left([u]^{\alpha} \cdot\left(\left(z_{l}(\alpha)-z_{r}(\alpha)\right) x+z_{r}(\alpha)\right), M^{\prime} M\right)}{\sup _{\alpha \in[0,1]} D_{I}^{(p)}\left([v]^{\alpha} \cdot\left(\left(z_{l}(\alpha)-z_{r}(\alpha)\right) x+z_{r}(\alpha)\right), M^{\prime} M\right)} \geq 1$
However,

$$
\begin{aligned}
& \frac{\sup _{\alpha \in[0,1]} D_{I}^{(p)}\left([u]^{\alpha} \cdot\left(\left(z_{l}(\alpha)-z_{r}(\alpha)\right) x+z_{r}(\alpha)\right), M^{\prime} M\right)}{\sup _{\alpha \in[0,1]} D_{I}^{(p)}\left([v]^{\alpha} \cdot\left(\left(z_{l}(\alpha)-z_{r}(\alpha)\right) x+z_{r}(\alpha)\right), M^{\prime} M\right)} \\
& =\frac{\sup _{\alpha \in[0,1]} D_{I}^{(p)}\left([u]^{\alpha} \cdot \frac{\left(\left(z_{l}(\alpha)-z_{r}(\alpha)\right) x+z_{r}(\alpha)\right)}{M^{\prime}}, M\right)}{\sup _{\alpha \in[0,1]} D_{I}^{(p)}\left([v]^{\alpha} \cdot \frac{\left(\left(z_{l}(\alpha)-z_{r}(\alpha)\right) x+z_{r}(\alpha)\right)}{M^{\prime}}, M\right)} \\
& \text { If } \\
& \qquad k(\alpha, x)=\frac{\left(\left(z_{l}(\alpha)-z_{r}(\alpha)\right) x+z_{r}(\alpha)\right)}{M^{\prime}}
\end{aligned}
$$

then

$$
0<k(\alpha, x)<1
$$

hence

$$
\begin{aligned}
& \frac{\sup _{\alpha \in[0,1]} D_{I}^{(p)}\left([u]^{\alpha} \cdot \frac{\left(\left(z_{l}(\alpha)-z_{r}(\alpha)\right) x+z_{r}(\alpha)\right)}{\left.M^{\prime}\right)}, M\right)}{\sup _{\alpha \in[0,1]} D_{I}^{(p)}\left([v]^{\alpha} \cdot \frac{\left(z_{l}(\alpha)-z_{r}(\alpha) x+z_{r}(\alpha)\right)}{M^{\prime}}, M\right)}= \\
& \frac{\sup _{\alpha \in[0,1]} D_{I}^{(p)}\left([u]^{\alpha} \cdot k(\alpha, x), M\right)}{\sup _{\alpha \in[0,1]} D_{I}^{(p)}\left([v]^{\alpha} \cdot k(\alpha, x), M\right)}= \\
& \frac{\sup _{\alpha \in[0,1]} k(\alpha, x) D_{I}^{(p)}\left([u]^{\alpha}, \frac{M}{k(\alpha, x)}\right)}{\sup _{\alpha \in[0,1]} k(\alpha, x) D_{I}^{(p)}\left([v]^{\alpha}, \frac{\alpha}{k(\alpha, x)}\right)} \geq \\
& \frac{\sup _{\alpha \in[0,1]} k(\alpha, x) D_{I}^{(p)}\left([u]^{\alpha}, T\right)}{\sup _{\alpha \in[0,1]} k(\alpha, x) D_{I}^{(p)}\left([u]^{\alpha}, T\right)}=1
\end{aligned}
$$

Because, regarding the assumption, $\tilde{u} \preceq \tilde{v}$ implies

$$
\frac{\sup _{\alpha \in[0,1]} D_{I}^{(p)}\left([u]^{\alpha}, M\right)}{\sup _{\alpha \in[0,1]} D_{I}^{(p)}\left([v]^{\alpha}, M\right)} \geq 1
$$

with $M \geq \max ($ supp $\tilde{u} \cup$ supp $\tilde{v})$. Therefore

$$
\frac{D_{F}^{(p)}\left(\tilde{u} \tilde{z}, M^{\prime} M\right)}{D_{F}^{(p)}\left(\tilde{v} \tilde{z}, M^{\prime} M\right)} \geq 1
$$

or

$$
D_{F}^{(p)}\left(\tilde{u} \tilde{z}, M^{\prime} M\right) \geq D_{F}^{(p)}\left(\tilde{v} \tilde{z}, M^{\prime} M\right)
$$

$\operatorname{Remark} 1$. If $\tilde{u} \precsim \tilde{v}$ then $-\tilde{u} \succsim-\tilde{v}$.

\section{Numerical example}

The results of comparing our proposed method with some other ranking methods ([16]) are summed up in Figure 1 and Table 1.

Figure 1: Figures of ranking.

Regarding to Figure 1 and Table 1 we have:

(a): $A_{1}=(.4, .9,1), A_{2}=(.4, .7,1), A_{3}=(.4, .5,1)$ then

$$
A_{3} \precsim A_{2} \precsim A_{1}
$$

(b): $A_{1}=(.2, .5, .8), A_{2}=(.4, .5, .6)$ then

$$
A_{1} \approx A_{2}
$$




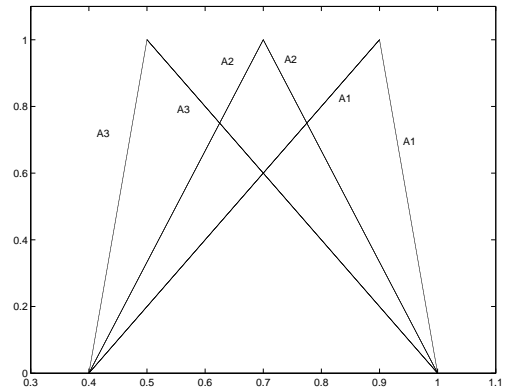

Figure 1: $\left(\tilde{A}_{1}, \tilde{A}_{2}, \tilde{A}_{3}\right)$

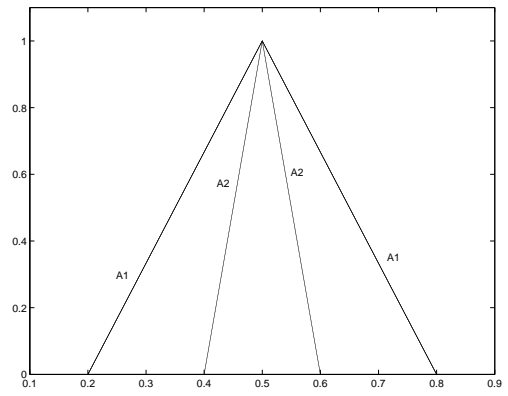

Figure 2: $\left(\tilde{A}_{1}, \tilde{A}_{2}\right)$

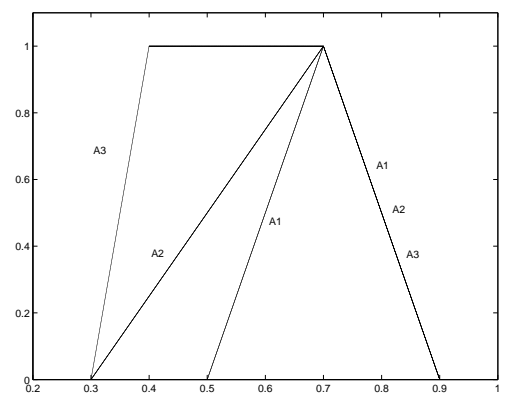

Figure 3: $\left(\left(\tilde{A}_{1}, \tilde{A}_{2}, \tilde{A}_{3}\right)\right.$



Figure 4: $\left(\tilde{A}_{1}, \tilde{A}_{2}, \tilde{A}_{3}\right)$

(c): $A_{1}=(.5, .7, .9), A_{2}=(.3, .7, .9), A_{3}=$ $(.3, .4, .7, .9)$ then

$$
A_{3} \precsim A_{2} \precsim A_{1}
$$

(d): $A_{1}=(.3, .5, .8, .9), A_{2}=(.3, .5, .9), A_{3}=$ $(.3, .5, .7)$ then

$$
A_{3} \precsim A_{2} \precsim A_{1}
$$

(e): $A_{1}=(.3, .3,1), A_{2}=(.1, .8, .8)$ then

$$
A_{1} \precsim A_{2}
$$



Figure 5: $\left(\tilde{A}_{1}, \tilde{A}_{2}\right)$

\section{Conclusions}

In this paper, a metric distance on interval numbers was introduced using the $\alpha$ - cut concept. Subsequently, it was extended to fuzzy numbers. Comparison of fuzzy numbers was made based on the degree of distance between the fuzzy numbers and crisp $\max (M)$ and crisp $\min (m)$. If we add a fuzzy number to the set of fuzzy numbers, then $M$ and $m$, as well as, may change, but there will be no changes in the ranking, which is the very useful property in the industrial problems. By some numerical examples, it was shown that the proposed method is more consistent with institution than the previous ranking methods.

\section{References}

[1] A. Bardossy and L. Duckstein, Fuzzy Rule-Based Modeling with Applications to Geophysical, Biological and Engineering Systems, CRC press, Boe, Raton, 1995.

[2] G. Bortolan and R. Degan. A review of some methods for ranking fuzzy sets, Fuzzy Sets and Systems, 15: 1-19, 1985.

[3] S.L. Chang and L.A. Zadeh, On fuzzy mapping and control, IEEE Trans. Syst. Man Cybernet. 2: 30-34, 1972.

[4] C.H. Cheng, A new approach for ranking fuzzy numbers by distance method, Fuzzy Sets and Systems, 95: 307-317, 1998.

[5] P. Diamond, Fuzzy least squares, Information Sciences 46: 141-157, 1988.

[6] P. Diamond and R. Korner, Extended fuzzy linear models and least squares estimates, Comput. Math. Appl. 33: 15-32, 1997.

[7] P. Diamond and H. Tanaka, Fuzzy regression analysis, in: R. Slowinski (Ed), Fuzzy Sets in Decision Analysis, Operations Research and Statistics, Kluwer Academic Publishers, Boston, 1998.

[8] D. Dubois and H. Prade, Fuzzy Sets and Systems: Theory and Applications, Academic Press, New York , 1980.

[9] P. Fortemps and M. Roubens, Ranking and defuzzification methods based on area compensation, Fuzzy Sets and Systems, 82: 319-330, 1996. 
[10] R. Goetschel and W. Vaxman, A pseudometric for fuzzy sets and certain related result, J. Math. Anal. Appl. 81: 507-523, 1981.

[11] R. Goetschel and W. Vaxman, Topological properties of fuzzy numbers, Fuzzy Sets and Systems, 10: 87-99, 1983.

[12] M. Ma, A. Kandel and M. Friedman, A new approach for defuzzification, Fuzzy Sets and Systems, 111: 351-356, 2000.

[13] M. Ma, A. Kandel and M. Friedman, Correction to "A new approach for defuzzification", Fuzzy Sets and Systems, 128: 133-134, 2000.

[14] M. Modarres and S.S. Nezhad, Ranking fuzzy numbers by pref erence ratio, Fuzzy Sets and Systems, 118: 429-439, 2000.

[15] T. Chu and C. Tsao, Ranking fuzzy numbers with an area between the centroid point and original point, Comput. Math. Appl., 43: 111$117,2002$.

[16] L. Tran and L. Duckstein, Comparison of fuzzy numbers using a fuzzy distance measure, Fuzzy Sets and Systems, 130: 331-341, 2002.

[17] X. Wang and E.E. Kerre, Reasonable properties for the ordering of fuzzy quantities I, Fuzzy Sets and Systems, 118: 375-385, 2001.

[18] R. Xu and C. Li, Multidimensional leastsquares fitting with a fuzzy model, Fuzzy Sets and Systems, 119: 215-223, 2001.

[19] J.S. Yao and K. Wu, Ranking fuzzy numbers based on decomposition principle and signed distance, Fuzzy Sets and Systems, 116: 275-288, 2000.

[20] Ze-Shui Xu and Jian Chen, An iteractive method for fuzzy multiple attribute group decision making, Information Sciences, 177: 248263,2006
Table 1: Comparison of fuzzy numbers by some methods.

\begin{tabular}{|c|c|c|c|c|c|c|}
\hline Methods & & & $a$ & & $b$ & \\
\hline & & $\overline{A_{1}}$ & $A_{2}$ & $A_{3}$ & $\overline{A_{1}}$ & $A_{2}$ \\
\hline \multirow[t]{3}{*}{ Yager } & $\overline{F_{1}}$ & 0.760 & 0.700 & 0.630 & 0.500 & 0.500 \\
\hline & $F_{2}$ & 0.900 & 0.760 & 0.660 & 0.610 & 0.540 \\
\hline & $F_{3}$ & 0.800 & 0.700 & 0.600 & 0.600 & 0.500 \\
\hline$B a-K w$ & & 1.000 & 0.740 & 0.600 & 1.000 & 1.000 \\
\hline \multirow[t]{3}{*}{ Bald-Gui } & $1: P$ & 0.420 & 0.330 & 0.330 & 0.270 & 0.270 \\
\hline & $g$ & 0.550 & 0.400 & 0.340 & 0.300 & 0.240 \\
\hline & $r: a$ & 0.280 & 0.230 & 0.220 & 0.200 & 0.230 \\
\hline Kerre & & 1.000 & 0.860 & 0.760 & 0.910 & 0.910 \\
\hline \multirow{3}{*}{ Jain } & $k=1$ & 0.900 & 0.760 & 0.660 & 0.730 & 0.670 \\
\hline & $k=2$ & 0.840 & 0.650 & 0.540 & 0.600 & 0.480 \\
\hline & $k=1 / 2$ & 0.950 & 0.860 & 0.780 & 0.830 & 0.800 \\
\hline \multirow[t]{4}{*}{$D u b-\operatorname{Pr} a$} & $P D$ & 1.000 & 0.740 & 0.600 & 1.000 & 1.000 \\
\hline & $P S D$ & 0.740 & 0.230 & 0.160 & 0.730 & 0.240 \\
\hline & $N D$ & 0.630 & 0.380 & 0.180 & 0.270 & 0.760 \\
\hline & $N S D$ & 0.260 & 0.000 & 0.000 & 0.000 & 0.000 \\
\hline \multirow[t]{4}{*}{$L e e-L i$} & U.m & 0.760 & 0.700 & 0.630 & 0.500 & 0.500 \\
\hline & $U . G$ & $\ldots$ & $\ldots$ & $\ldots$ & 0.120 & 0.040 \\
\hline & P.m & 0.800 & 0.700 & 0.600 & 0.500 & 0.500 \\
\hline & $P . G$ & $\ldots$ & $\ldots$ & $\ldots$ & 0.090 & 0.030 \\
\hline For - Rou & $\overline{F_{0}}$ & 0.800 & 0.700 & 0.600 & 0.500 & 0.500 \\
\hline \multirow[t]{4}{*}{ Tran -Duc } & $D_{\max } ; f=x$ & 0.187 & 0.308 & 0.442 & 0.505 & 0.501 \\
\hline & $D_{\min } ; f=x$ & 0.838 & 0.704 & 0.573 & 0.505 & 0.501 \\
\hline & $D_{\max } ; f=1$ & 0.231 & 0.316 & 0.416 & 0.510 & 0.501 \\
\hline & $D_{\min } ; f=1$ & 0.808 & 0.707 & 0.611 & 0.510 & 0.501 \\
\hline$\overline{d_{F}}$ & $\gamma_{d}^{(2)}(., M)$ & 0.409 & 0.500 & 0.591 & 0.500 & 0.500 \\
\hline$d_{F}$ & $\gamma_{d}^{(2)}(., m)$ & 0.591 & 0.500 & 0.409 & 0.500 & 0.500 \\
\hline
\end{tabular}

Table 1.(continued)

\begin{tabular}{|c|c|c|c|c|c|c|c|}
\hline & $c$ & & & $d$ & & $e$ & \\
\hline$A_{1}$ & $A_{2}$ & $A_{3}$ & $A_{1}$ & $A_{2}$ & $A_{3}$ & $A_{1}$ & $A_{2}$ \\
\hline 0.700 & 0.630 & 0.570 & 0.620 & 0.560 & 0.500 & 0.610 & 0.530 \\
\hline 0.750 & 0.750 & 0.750 & 0.810 & 0.640 & 0.580 & 0.660 & 0.690 \\
\hline 0.700 & 0.650 & 0.570 & 0.620 & 0.540 & 0.500 & 0.580 & 0.560 \\
\hline 1.000 & 1.000 & 1.000 & 1.000 & 1.000 & 1.000 & 0.840 & 1.000 \\
\hline 0.370 & 0.270 & 0.270 & 0.450 & 0.370 & 0.270 & 0.420 & 0.330 \\
\hline 0.420 & 0.350 & 0.350 & 0.350 & 0.400 & 0.280 & 0.440 & 0.370 \\
\hline 0.270 & 0.190 & 0.190 & 0.310 & 0.280 & 0.210 & 0.340 & 0.240 \\
\hline 1.000 & 0.910 & 0.750 & 1.000 & 0.850 & 0.750 & 0.960 & 0.890 \\
\hline 0.820 & 0.820 & 0.820 & 0.900 & 0.690 & 0.640 & 0.660 & 0.690 \\
\hline 0.710 & 0.710 & 0.710 & 0.820 & 0.560 & 0.450 & 0.530 & 0.510 \\
\hline 0.890 & 0.890 & 0.890 & 0.940 & 0.800 & 0.770 & 0.780 & 0.810 \\
\hline 1.000 & 1.000 & 1.000 & 1.000 & 1.000 & 1.000 & 0.840 & 1.000 \\
\hline 0.500 & 0.500 & 0.500 & 0.800 & 0.200 & 0.000 & 0.540 & 0.460 \\
\hline 0.670 & 0.350 & 0.000 & 0.500 & 0.500 & 0.500 & 0.540 & 0.460 \\
\hline 0.000 & 0.000 & 0.000 & 0.000 & 0.000 & 0.000 & 0.000 & 0.160 \\
\hline 0.700 & 0.630 & 0.570 & 0.620 & 0.560 & 0.500 & 0.610 & 0.530 \\
\hline$\ldots$ & $\ldots$ & $\ldots$ & $\ldots$ & $\ldots$ & $\ldots$ & $\ldots$ & $\ldots$ \\
\hline 0.700 & 0.650 & 0.580 & 0.630 & 0.550 & 0.500 & 0.530 & 0.580 \\
\hline$\ldots$ & $\ldots$ & $\ldots$ & $\ldots$ & $\ldots$ & $\ldots$ & $\ldots$ & $\ldots$ \\
\hline 0.700 & 0.650 & 0.575 & 0.625 & 0.550 & 0.500 & 0.490 & 0.610 \\
\hline 0.304 & 0.342 & 0.457 & 0.395 & 0.473 & 0.502 & 0.574 & 0.355 \\
\hline 0.702 & 0.671 & 0.585 & 0.650 & 0.539 & 0.502 & 0.451 & 0.673 \\
\hline 0.307 & 0.365 & 0.445 & 0.398 & 0.462 & 0.504 & 0.531 & 0.417 \\
\hline 0.703 & 0.658 & 0.590 & 0.639 & 0.560 & 0.504 & 0.512 & 0.628 \\
\hline 0.357 & 0.464 & 0.510 & 0.522 & 0.536 & 0.643 & 0.544 & 0.453 \\
\hline 0.643 & 0.536 & 0.490 & 0.478 & 0.464 & 0.357 & 0.456 & 0.547 \\
\hline
\end{tabular}

